\title{
Synthesizing tungsten oxide nanowires by a thermal evaporation method
}

\author{
Kunquan Hong, Maohai Xie, ${ }^{\text {a) }}$ Rong Hu, and Huasheng Wu \\ Physics Department, The University of Hong Kong, Pokfulam Road, Hong Kong, People's Republic of \\ China and the HKU-CAS Joint Laboratory on New Materials, The University of Hong Kong, Pokfulam Road, \\ Hong Kong, People's Republic of China
}

(Received 19 January 2007; accepted 3 April 2007; published online 26 April 2007)

\begin{abstract}
Tungsten oxide $\mathrm{W}_{18} \mathrm{O}_{49}$ nanowires with diameters of $10-20 \mathrm{~nm}$ were synthesized with high yield by thermal evaporation in a tube furnace. By heating tungsten trioxide powder at $900{ }^{\circ} \mathrm{C}$ in vacuum $\left(5 \times 10^{-3}\right.$ torr $), \mathrm{W}_{18} \mathrm{O}_{49}$ nanowires with diameters of $10-20 \mathrm{~nm}$ and lengths up to micrometers were produced with high yield on the Au-coated $\mathrm{Si}$ substrates located in the low temperature zone $\left(550-600{ }^{\circ} \mathrm{C}\right)$ of the furnace. The morphology, composition, and crystal structure of the nanowires were characterized by various methods. The conditions and the mechanism of $\mathrm{W}_{18} \mathrm{O}_{49}$ nanowire growth are discussed. (C) 2007 American Institute of Physics. [DOI: 10.1063/1.2734175]
\end{abstract}

Semiconductor nanowires have attracted much attention in the past years for their potentials in future nanodevice applications. ${ }^{1-3}$ Many methods have been developed to synthesize one dimensional nanostructured materials, such as wet chemical reaction, ${ }^{4}$ thermal evaporation, ${ }^{5}$ laser ablation, ${ }^{6}$ sol-gel and template directed synthesis, ${ }^{7}$ etc. The method of thermal evaporation has the advantage of being simple, cheap, and flexible. It has been widely used to synthesize various semiconductor nanowires. Among these semiconductors, tungsten oxide is of great interest for its outstanding chromic properties and thus for its optical and sensor applications. Tungsten oxide in the bulk form has been extensively investigated in the past decades, ${ }^{8-10}$ while a few methods have also been developed to synthesize its nanostructures. ${ }^{11-17}$ Generally, a high temperature $\left(1200-2000{ }^{\circ} \mathrm{C}\right)$ is required for synthesizing tungsten oxide nanostructures, ${ }^{11-14}$ and the experimental equipments are also rather complex and demanding. In this letter, we report a simple thermal evaporation method for fabricating tungsten oxide nanowires on $\mathrm{Si}$ wafers. By evaporating tungsten trioxide powder in a tube furnace at $900{ }^{\circ} \mathrm{C}$, tungsten oxide nanowires with diameters of $10-20 \mathrm{~nm}$ and lengths up to several micrometers are obtained with high yield on the Aucoated $\mathrm{Si}$ substrate located in the low temperature zone of $550-600{ }^{\circ} \mathrm{C}$.

The nanowire growth experiments were carried out in a conventional horizontal tube furnace with a quartz working tube. ${ }^{15}$ Tungsten trioxide powder $(0.5 \mathrm{~g}$, Fluka, $99.9 \%$ in purity) was deposited on a quartz boat and placed in the uniform-temperature zone of the tube furnace, which acted as the source material. A silicon (100) wafer coated with a thin layer of $\mathrm{Au}$ (thickness of about $5 \mathrm{~nm}$ ) by sputter coating was placed in the low temperature zone, $\sim 13 \mathrm{~cm}$ downstream the source and acted as the substrate. After the quartz tube was pumped to the desired vacuum of $(5-7)$ $\times 10^{-3}$ torr torr with an air flow rate of $\sim 0.13 \mathrm{SCCM}$ (SCCM denotes cubic centimeter per minute at STP), the temperature of the furnace was raised from room temperature (RT) to $900{ }^{\circ} \mathrm{C}$ at a ramping rate of $30{ }^{\circ} \mathrm{C} / \mathrm{min}$. The temperature of the substrate was increased concurrently to $550-600{ }^{\circ} \mathrm{C}$. After maintaining the high temperature for $3 \mathrm{~h}$,

a) Author to whom correspondence should be addressed: FAX: 0085225599152; electronic mail: mhxie@hkusua.hku.hk the furnace was let cool naturally to room temperature before taking out the sample for characterization.

The structural, morphological, and compositional properties of the samples were characterized by using scanning electron microscopy (SEM) (LEO1530), x-ray diffraction (XRD) (Huber V612365), and high resolution transmission electron microscopy (HRTEM) (Tecnai G2-20 scanning transmission electron microscope). Selected area electron diffraction (SAED) and energy-dispersive x-ray (EDX) spectroscopy were also conducted during the TEM experiments. Photoluminescence (PL) measurements of the sample were carried out at room temperature using a $\mathrm{He}-\mathrm{Cd}$ laser (wavelength of $325 \mathrm{~nm}$ ) as the excitation source. The signals were dispersed by a Spex $750 \mathrm{M}$ monochromator and detected by a photomultiplier.

Figures 1(a)-1(d) show a series of SEM images at different magnifications for a typical sample, depicting the morphology of the resulted tungsten oxide nanowires grown on the silicon substrate. The product is seen to consist of a pure wirelike structure with high density and at large scale. These nanowires are quite uniform in diameter, ranging from 10 to $20 \mathrm{~nm}$, and up to several micrometers long.

The XRD spectrum of the sample [Fig. 2(a)] shows that the reflection peaks can well be indexed as the reflections
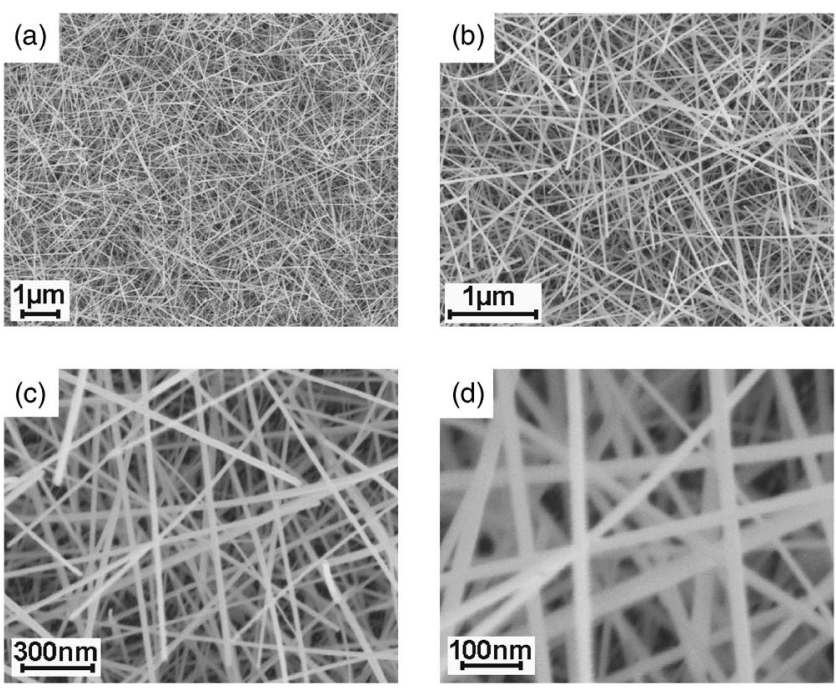

FIG. 1. SEM images of a sample at different magnifications. 

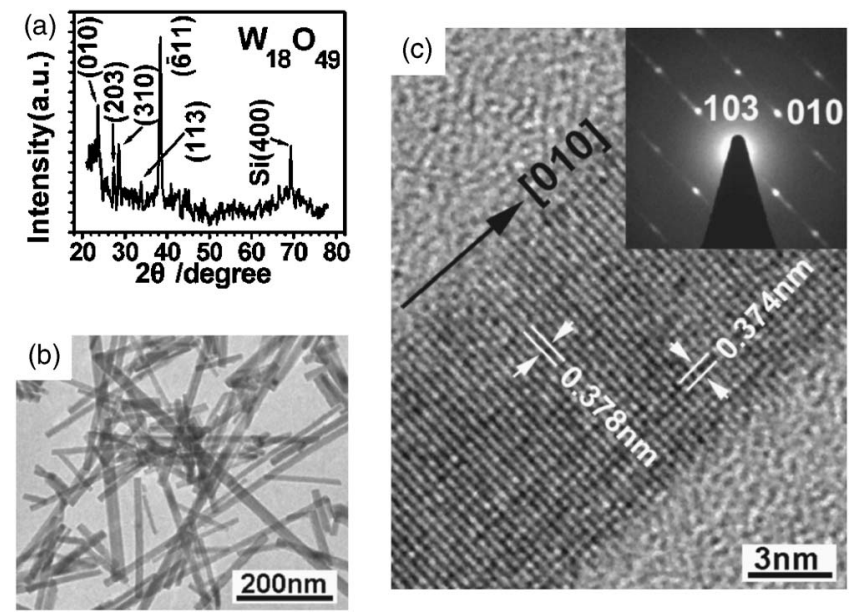

FIG. 2. (a) XRD spectrum of the sample; (b) TEM image and (c) HRTEM image of the nanowire. The inset presents the SAED pattern of the nanowire.

from planes of monoclinic $\mathrm{W}_{18} \mathrm{O}_{49}$ ( $\gamma$-tungsten oxide), with $\beta=115.20^{\circ}, \quad a=1.8334 \mathrm{~nm}, \quad b=0.3786 \mathrm{~nm}, \quad$ and $c=1.4044 \mathrm{~nm}^{18}$ No diffraction peaks from Au or other impurities were detected. The morphology and structure of the nanowires were further studied by TEM. Figures 2(b) and 2(c) present the TEM image and high resolution TEM image of the nanowire, respectively, while the corresponding selected area electron diffraction pattern is given in the inset. The lattice spacing are measured to be 0.378 and $0.374 \mathrm{~nm}$ along the two orthonormal directions, corresponding to the (010) and (103) planes of monoclinic $\mathrm{W}_{18} \mathrm{O}_{49}$. It is shown that the [010] is the major growth direction of the nanowire, as also confirmed by the SAED patterns. The EDX analysis of the sample further reveals that the sample contains $\mathrm{W}$ and $\mathrm{O}$ only, with the molar ratio of $\mathrm{W}: \mathrm{O}=1: 2.77$, which is consistent with the XRD result of it being $\mathrm{W}_{18} \mathrm{O}_{49}\left(\mathrm{WO}_{2.72}\right)$.

Our experiments suggest that the precoated $\mathrm{Au}$ film on the Si substrate plays a very important role in the growth of tungsten oxide nanowires under such a growth condition. Figure 3(a) shows a SEM image of a sample synthesized using the same method and conditions as that for growing the sample of Fig. 1, except that there are lines free of Au on the silicon surface. As is seen, the same tungsten oxide nanowires are produced in regions where there exist $\mathrm{Au}$ films [Figs. 3(a) and 3(b)], similar to the case of Fig. 1, but there
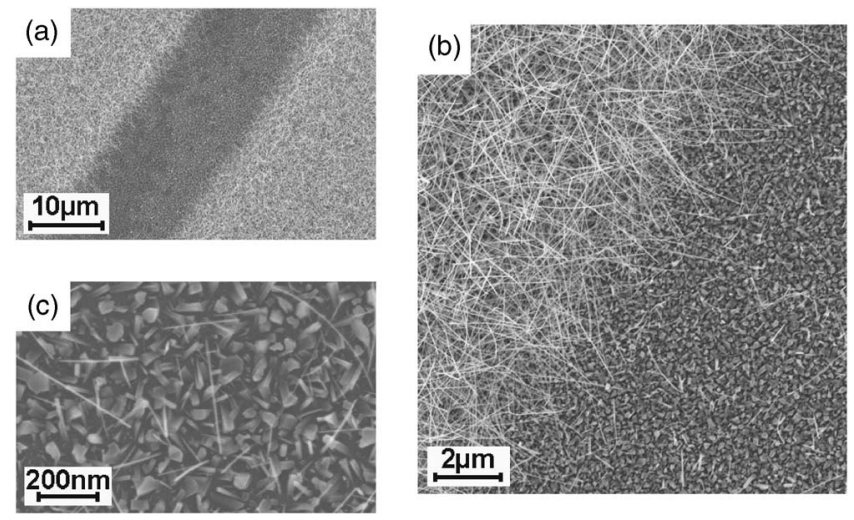

FIG. 3. (a) SEM images of the sample with a line free of Au film and (b) the enlarged image depicting the boundary between regions with and without $\mathrm{Au}(\mathrm{c})$ shows the region free of $\mathrm{Au}$.
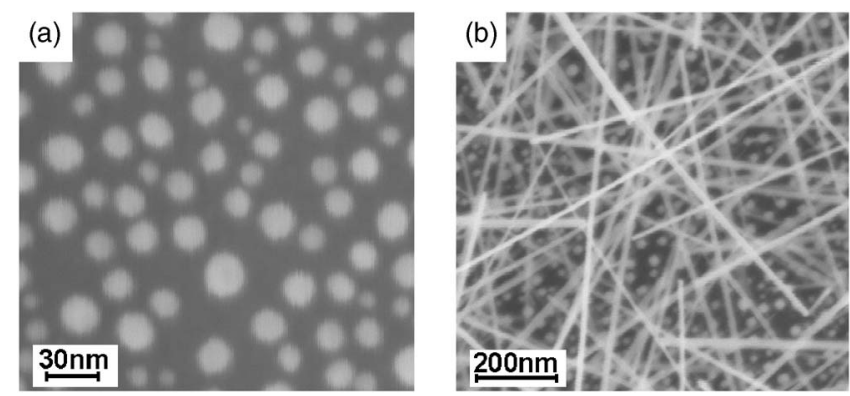

FIG. 4. SEM images of samples prepared under the pressures (a) 3 $\times 10^{-2}$ torr and (b) $1 \times 10^{-2}$ torr.

are few nanowires in regions without the Au. Instead, nanoparticles with diameters of $\sim 50 \mathrm{~nm}$ are seen in these latter regions [Fig. 3(c)]. The boundary between the regions with and without the Au film is obvious and sharp [Fig. 3(b)]. These findings unambiguously demonstrate the important roles played by $\mathrm{Au}$ in tungsten oxide nanowire growth on Si.

To further reveal the necessary conditions for the $\mathrm{W}_{18} \mathrm{O}_{49}$ nanowire growth, we have also prepared samples using the same method but changing the growth pressure. Figures 4(a) and 4(b) show SEM images of the resulted samples grown at $3 \times 10^{-2}$ and $1 \times 10^{-2}$ torr, respectively. In Fig. 4(a), there is no nanowire but lots of $\mathrm{Au}$ nanoparticles on the substrate. Though tungsten oxide nanowire can grow at $1 \times 10^{-2}$ torr, the yield is still low and the Au clusters remain visible on the surface of silicon wafer [Fig. 4(b)]. For growth under a higher vacuum [better than (2-3) $\times 10^{-3}$ torr], we start to observe nanowire formation on bare $\mathrm{Si}(100){ }^{19}$ This latter result seems to be consistent with the literature report that tungsten oxide nanowires grow on indiumtin oxide under vacuum of $10^{-5}-10^{-4}$ torr, ${ }^{16}$ or on $\mathrm{Si}(111)$ surface under vacuum of $(2-3) \times 10^{-3}$ torr $^{17}$ even without the Au catalyst.

In a typical vapor-liquid-solid (VLS) growth of nanowires, $\mathrm{Au}$ nanoparticles are usually found at the top or bottom of the nanowires. ${ }^{2,20}$ However, for tungsten oxide nanowires synthesized in this study, no such relation is found. So the growth mechanism is unlikely to be the typical VLS mechanism. Instead, the vapor-solid mechanism is likely responsible for the tungsten oxide nanowire growth in this experiment. Tungsten trioxide begins to sublimate from the quartz boat when the temperature is increased to $750{ }^{\circ} \mathrm{C}$, and the process is greatly enhanced at the temperature of $900{ }^{\circ} \mathrm{C}$. The sublimated tungsten trioxide vapor flows to the lower temperature zone where the substrate is placed and becomes supersaturated for nucleation of small clusters and for subsequent growth of nanowires. When the vacuum of the furnace is not so high, the mean free path of the vapor is small, which affects the degree of supersaturation over the substrate and thus hinders the nucleation process. When the vacuum becomes significantly higher so that the mean free path of the tungsten trioxide vapor is greatly enhanced, its partial pressure, and thus the supersaturation, is increased in the substrate zone, which enhances nucleation of tungsten oxide on substrate wafers. ${ }^{21}$ For the furnace with intermediate vacuum, nucleation on a bare Si surface is rare but on the Au-coated substrate, nucleation is promoted by modifying the adsorption and diffusion characteristics of the surface. The diffusivity of atoms/molecules on Au-coated $\mathrm{Si}$ is expected to be different from that on bare $\mathrm{Si}$, so for a given 


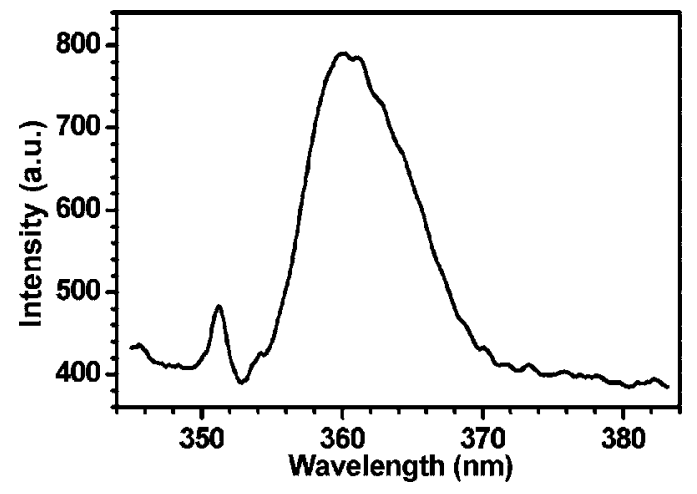

FIG. 5. Room temperature PL spectrum of the tungsten oxide nanowires.

concentration of adsorbate, the nucleation rate is different. With the presence of $\mathrm{Au}$, the atoms/molecules are suspected to be preferentially adsorbed and trapped on such substrate. The increased concentration of adsorbates on such substrate makes nucleation more efficient, promoting the nanowire growth. Further evidence showing many tungsten oxide nanowires growing out of single Au cluster under similar growth conditions exist. ${ }^{19}$

Finally, the optical properties of the synthesized tungsten oxide nanowires are characterized by room temperature PL. Figure 5 shows a typical PL spectrum, revealing the dominant luminescence peaks at 351 and $361 \mathrm{~nm}$. Tungsten trioxide is an indirect band gap semiconductor, and its crystals or thin films do not give rise to such strong luminescence. ${ }^{17}$ Similar PL peaks (at $355 \mathrm{~nm}$ ) have been detected by Feng et $a l$. for thermally evaporated $\mathrm{W}_{18} \mathrm{O}_{49}$ nanowires, and they attributed the strong luminescence to the quantum effect. ${ }^{17} \mathrm{On}$ the other hand, other origins such as native or foreign defects in the structures cannot be ruled out.

In summary, we show the synthesis of tungsten oxide nanowires on silicon wafer with large scale and high yield. The nanowires are quite uniform in diameters, within a range of $10-20 \mathrm{~nm}$, and lengths up to a few micrometers. The effects of working pressure of the furnace as well as that of predeposited Au film are examined. The likely growth mechanism has also been discussed.

The authors would like to thank J. Q. Ning and S. J. Xu for taking the PL measurements. This work is financially supported by grants from the Research Grant Council of the Hong Kong Special Administrative Region, China under Grant Nos. HKU 7047/05P and HKU 7046/05P.

${ }^{1}$ J. S. Jie, W. J. Zhang, Y. Jiang, X. M. Meng, Y. Q. Li, and S. T. Lee, Nano Lett. 6, 1887 (2006).

${ }^{2}$ M. H. Huang, S. Mao, H. Feick, H. Q. Yan, Y. Y. Wu, H. Kind, E. Weber, R. Russo, and P. D. Yang, Science 292, 1897 (2001).

${ }^{3}$ R. J. Chen, N. R. Franklin, J. Kong, J. Cao, T. W. Tombler, Y. G. Zhang, and H. J. Dai, Appl. Phys. Lett. 79, 2258 (2001).

${ }^{4}$ Y. K. Liu, C. L. Zheng, W. Z. Wang, Y. J. Zhan, and G. H. Wang, J. Cryst. Growth 233, 8 (2001).

${ }^{5}$ Z. W. Pan, Z. R. Dai, and Z. L. Wang, Science 291, 1947 (2001).

${ }^{6}$ Y. K. Liu, J. A. Zapien, Y. Y. Shan, C. Y. Geng, C. S. Lee, and S. T. Lee, Adv. Mater. (Weinheim, Ger.) 17, 1372 (2005).

${ }^{7}$ L. F. Cheng, X. T. Zhang, B. Liu, H. Z. Wang, Y. C. Li, Y. B. Huang, and Z. L. Du, Nanotechnology 16, 1341 (2005).

${ }^{8}$ J. L. Solis, S. Saukko, L. Kish, C. G. Granqvist, and V. Lantto, Thin Solid Films 391, 255 (2001).

${ }^{9}$ O. Bohnke, M. Rezrazi, B. Vuillemin, C. Bohnke, P. A. Gillet, and C. Rousselot, Sol. Energy Mater. Sol. Cells 25, 361 (1992).

${ }^{10}$ C. Santato, M. Odziemkowski, M. Ulmann, and J. Augustynski, J. Am. Chem. Soc. 123, 10639 (2001).

${ }^{11}$ Y. Wu, Z. H. Xi, G. M. Zhang, J. Yu, and D. Z. Guo, J. Cryst. Growth 292, 143 (2006)

${ }^{12}$ Z. W. Liu, Y. S. Bando, and C. C. Tang, Chem. Phys. Lett. 372, 179 (2003).

${ }^{13}$ A. C. Dillon, A. H. Mahan, R. Deshpande, J. L. Alleman, J. L. Blackbum, P. A. Parillia, M. J. Heben, C. Engtrakul, K. E. H. Gibert, K. M. Jounes, R. To, S. H. Lee, and J. H. Lehman, Thin Solid Films 501, 216 (2006).

${ }^{14}$ S. Vaddiraju, H. Chandrasekaran, and M. K. Sunkara, J. Am. Chem. Soc. 125, 10792 (2003).

${ }^{15}$ K. Q. Hong, M. H. Xie, and H. S. Wu, Nanotechnology 17, 4830 (2006).

${ }^{16}$ C. C. Liao, F. R. Chen, and J. J. Kai, Sol. Energy Mater. Sol. Cells 90, 1147 (2006)

${ }^{17}$ M. Feng, A. L. Pan, H. R. Zhang, Z. A. Li, F. Liu, H. W. Liu, D. X. Shi, B. S. Zou, and H. J. Gao, Appl. Phys. Lett. 86, 141901 (2005).

${ }^{18}$ JCPDS Card No. 71-2450.

${ }^{19}$ K. Q. Hong, M. H. Xie, R. Hu, and H. S. Wu (unpublished).

${ }^{20}$ X. F. Duan and C. M. Lieber, J. Am. Chem. Soc. 122, 188 (2000).

${ }^{21}$ Z. R. Dai, Z. W. Pan, and Z. L. Wang, Adv. Funct. Mater. 13, 9 (2003). 
Applied Physics Letters is copyrighted by the American Institute of Physics (AIP). Redistribution of journal material is subject to the AIP online journal license and/or AIP copyright. For more information, see http://ojps.aip.org/aplo/aplcr.jsp 